\title{
SingHealth Radiology Archives pictorial essay Part 1: cardiovascular, respiratory and neurological cases
}

\author{
Mark Bangwei Tan ${ }^{1}$, MRCS, FRCR, Kim Ping $\operatorname{Tan}^{1}$, FRCR, FAMS, Joey Chan Yiing Beh ${ }^{2}$, MMed, FRCR, \\ Eugenie Yi Kar $\underline{\mathrm{Chan}}^{3}$, MBBS, Kenneth Fu Wen $\underline{\mathrm{Chin}}^{3}$, MD, Zong Yi $\underline{\mathrm{Chin}}{ }^{3}$, MBChB, MRCS, Wei Ming $\underline{\mathrm{Chua}}{ }^{3}$, MMed, FRCR, \\ Aaron Wei-Loong Chong ${ }^{3}$, MB BCh BAO, Gary Tianyu $\mathrm{Gu}^{3}$, MBBS, MRCS, Wenlu $\mathrm{Hou}^{3}$, MMed, FRCR, \\ Anna Chooi Yan Lai ${ }^{1}$, MMed, FRCR, Rebekah Zhuyi Lee ${ }^{3}$, MBBS, Perry Jia Ren Liew ${ }^{4}$, MMed, FRCR, \\ May Yi Shan $\underline{L i m}^{3}$, MRCS, FRCR, Joshua Li Liang $\underline{\operatorname{Lim}}^{3}$, MBBS, Zehao $\underline{\operatorname{Tan}}^{3}$, MMed, FRCR, Eelin $\underline{\operatorname{Tan}}^{3}$, BMed, MD, \\ Grace Siew Lim $\operatorname{Tan}^{3}$, MBBS, Timothy Shao Ern $\underline{\operatorname{Tan}}^{3}, \mathrm{MBChB}, E$ U Jin $\underline{\operatorname{Tan}^{1}}$, MMed, FRCR, \\ Alexander Sheng Ming $\underline{\operatorname{Tan}}^{1}$, MRCS, FRCR, Yet Yen $\underline{\text { Yan }}^{4}$, MMed, FRCR, Winston Eng Hoe $\underline{\operatorname{Lim}}^{1}$, FRCR, FAMS
}

The Singapore Health Services cluster (SingHealth) radiology film archives are a valuable repository of local radiological cases dating back to the 1950s. Some of the cases in the archives are of historical medical interest, i.e. cerebral angiography in the workup of patients with hemiplegia. Other cases are of historical social interest, being conditions seen during earlier stages of Singapore's development, i.e. bound feet. The archives form a unique portal into the development of local radiology as well as the national development of Singapore. A selection from the archives is published in 2020 in commemoration of the 20th anniversary of the formation of SingHealth, the 55th National Day of Singapore, and the 125th anniversary of the International Day of Radiology. This pictorial essay comprises cardiovascular, respiratory and neurological cases from the archives.

Keywords: archives, historical, national development, pictorial essay, SingHealth

\section{INTRODUCTION}

The Singapore Health Services (SingHealth) radiology film archives comprise radiography case records dating back to the 1950s. The archives are located within Singapore General Hospital (SGH) and comprise cases of historical medical interest as well as of historical social interest. The archives form a unique portal into the development of local radiology as well as the national development of Singapore. Additional commentary and anecdotes on these cases were obtained via oral interview with Dr Tan Kim Ping, a prominent local radiologist, former head of department of the SGH Department of Radiology and key proponent of the archives. A selection from the archives was published in 2020 in commemoration of the 20th anniversary of the formation of SingHealth, the 55th National Day of Singapore, and the 125th anniversary of the International Day of Radiology. This pictorial essay comprises cardiovascular, respiratory and neurological cases from the archives.

\section{RADIOLOGIST STAFFING IN NOVEMBER 1976}

In the 1970s, nine radiologists provided fluoroscopy, interventional radiology and plain radiography reporting services to SGH and three satellite (outstation) hospitals - Thomson Road General Hospital (precursor of the current Changi General Hospital), Alexandra Hospital and Kandang Kerbau Maternity Hospital (renamed KK Women's and Children's Hospital in 1997). A single doctor would provide on-call services for these four hospitals.
A radiologist roster from November 1976 is shown in Fig. 1. Today, the radiological services of these same hospitals have expanded to include ultrasonography, computed tomography $(\mathrm{CT})$, magnetic resonance (MR) imaging and nuclear medicine investigations, provided by a staff of at least 150 radiologists and trainees.

\section{STANFORD TYPE A AORTIC DISSECTION}

Fig. 2 shows transfemoral catheter aortography that was performed for a patient with chest pain and unequal pulses. In the thoracic aorta, a dissection flap arising near the aortic root is visualised (black arrow, Fig. 2a). The false lumen is less dense (white arrows in Fig. 2). There is also non-opacification of the left subclavian artery, indicating its origin from the false lumen. As the dissection involves the ascending aorta proximal to the left subclavian artery, this is classified as a Stanford Type A aortic dissection. In the abdominal aorta, the presence of a right nephrogram (black arrow, Fig. 2b) without opacification of the left kidney and renal artery suggests that the latter structures arise from the false lumen.

Catheter aortography was previously the gold standard for suspected aortic dissection. One of its pitfalls, however, is a false negative due to thrombosis of the false lumen. ${ }^{(1)}$ CT has since replaced this procedure due to its high image resolution and amenability to post-processing reconstructions, as displayed in a three-dimensional reconstruction and

\footnotetext{
${ }^{1}$ Department of Diagnostic Radiology, Singapore General Hospital, ${ }^{2}$ Department of Radiology, Ng Teng Fong General Hospital, ${ }^{3}$ Singhealth Diagnostic Radiology Residency Programme, ${ }^{4}$ Department of Radiology, Changi General Hospital, Singapore

Correspondence: Dr Mark Tan Bangwei, Associate Consultant, Department of Diagnostic Radiology, Singapore General Hospital, Outram Road, Singapore 169608. marktanbangwei@gmail.com
} 


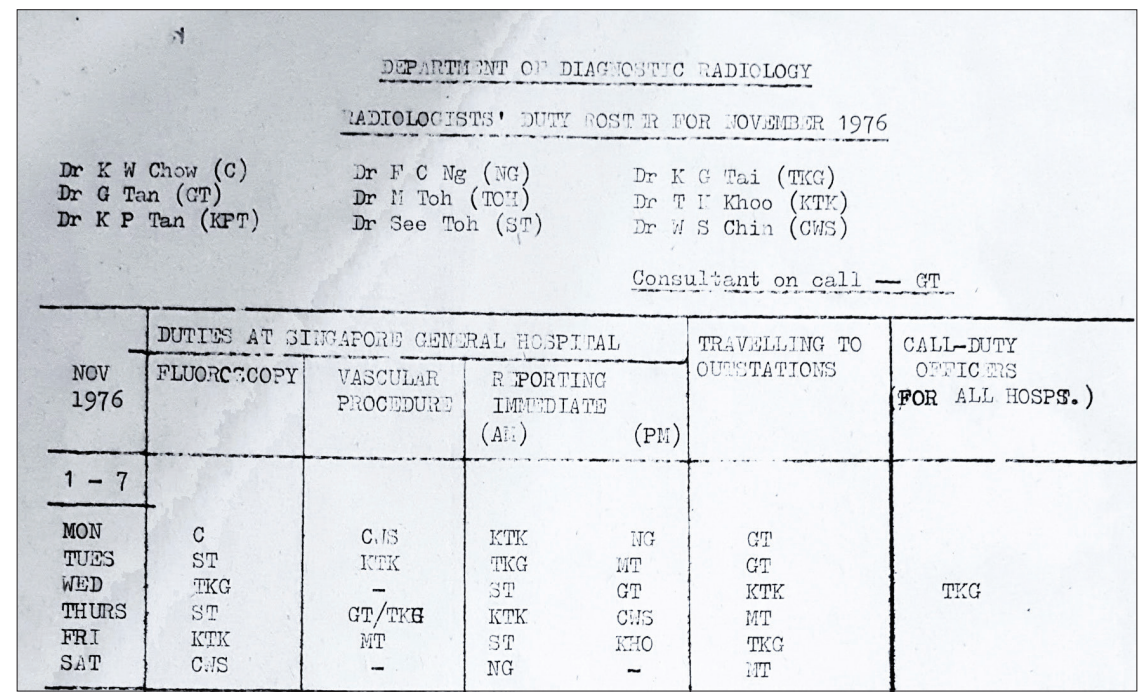

Fig. 1 Radiologist roster in November 1976.
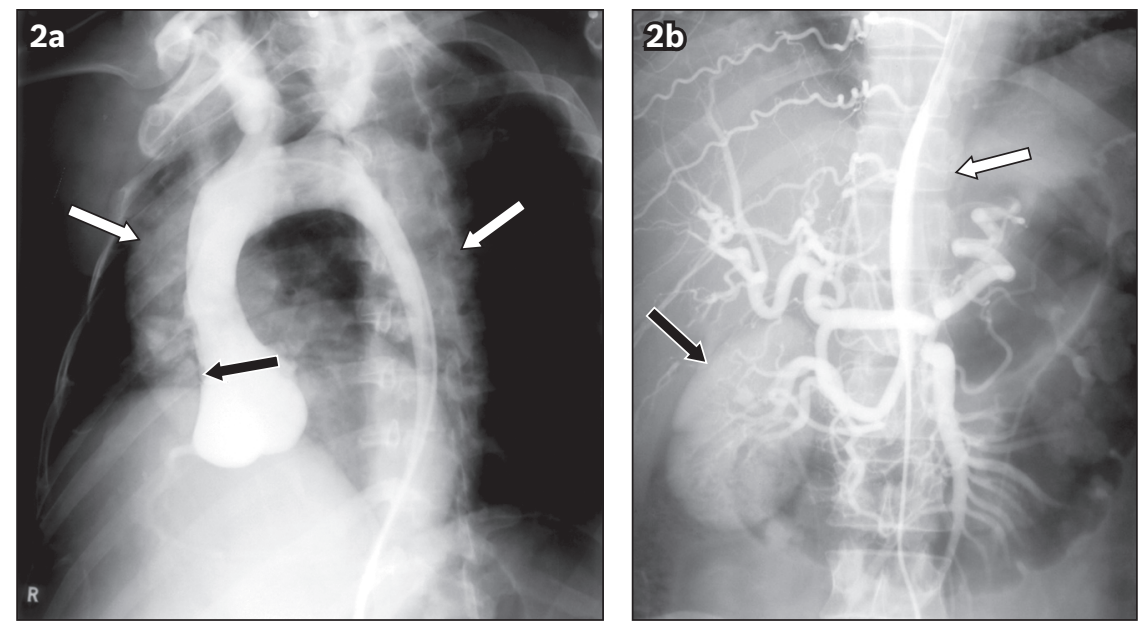

Fig. 2 Transfemoral catheter aortogram shows the (a) thoracic aorta and (b) abdominal aorta in the setting of a Stanford Type A aortic dissection

maximum intensity projection sagittal image (Fig. 3), whereby the dissection flap (arrows) and the subtle contrast differences between the true lumen (asterisks) and false lumen can be appreciated in exquisite detail. Nonetheless, the recognition of this appearance of aortic dissection on angiography remains relevant to this day, as it can occasionally be seen during modern procedures involving cannulation of the aorta (e.g. cardiac catheterisation, visceral embolisation). Notably, this study was performed at Tan Tock Seng Hospital (TTSH), which was previously the designated hospital for cardiothoracic surgery in Singapore.

\section{LEFT ATRIAL ENLARGEMENT ON BARIUM SWALLOW}

A middle-aged woman presented with hoarseness of voice. A barium swallow study performed in the lateral projection (Fig. 4) demonstrated posterior displacement of the distal oesophagus (arrows), which is a classic radiological finding of left atrial enlargement. ${ }^{(2)}$ The left atrium is the most posteriorly located cardiac chamber. It can become enlarged secondary to increased intra-atrial pressures, which may be seen in acquired conditions such as mitral valve disease, as well as in congenital conditions such as a ventricular septal defect or patent ductus arteriosus. Patients with left atrial enlargement may present with dysphagia to solids due to extrinsic compression of the oesophagus (dysphagia megalatriensis), as well as hoarseness of voice due to compression of the recurrent laryngeal nerve (Ortner syndrome). The barium swallow study was a commonly used surrogate method to diagnose left atrial enlargement before echocardiography (Fig. 5) became more widely available.

\section{EISENMENGER SYNDROME SECONDARY TO ATRIAL SEPTAL DEFECT}

Chest radiography was performed in a woman with chronic shortness of breath (Fig. 6). There is enlargement of the pulmonary trunk and pulmonary arteries with decreased size of the peripheral vessels (i.e. 'pruning'). This is suggestive of the presence of an atrial septal defect with imaging features of resultant pulmonary arterial hypertension. Some patients may develop shunt reversal from a left-to-right to a rightto-left direction as a result of chronic pulmonary arterial hypertension, leading to right ventricular hypertrophy and 

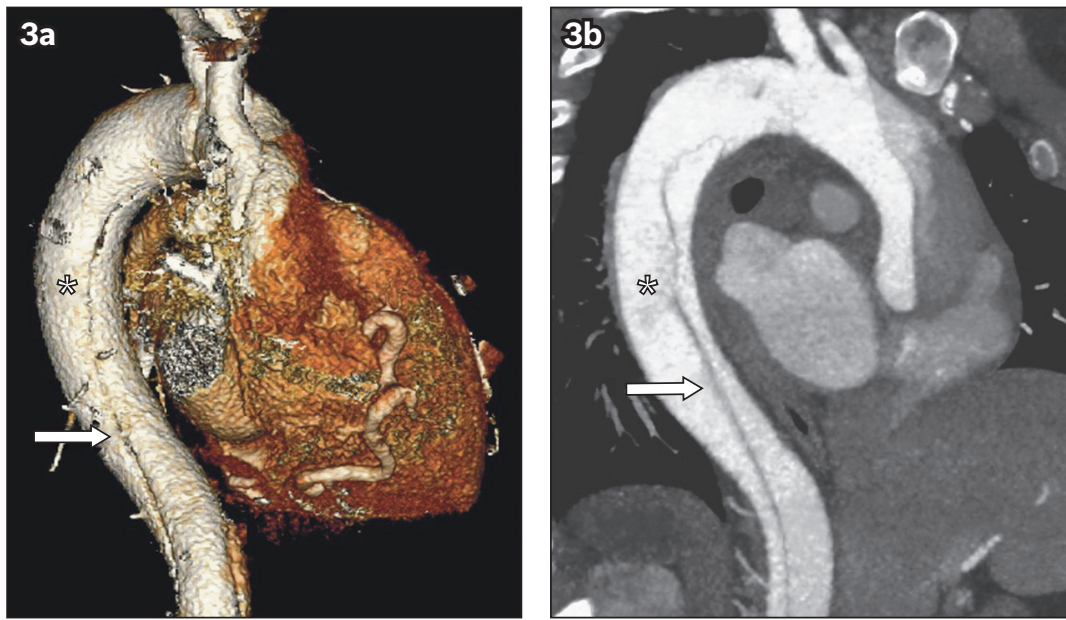

Fig. 3 (a) Three-dimensional reconstruction and (b) maximum intensity projection sagittal CT images show the dissection flap (arrows) and the subtle contrast differences between the true lumen (asterisks) and false lumen in the setting of a Stanford Type A aortic dissection.

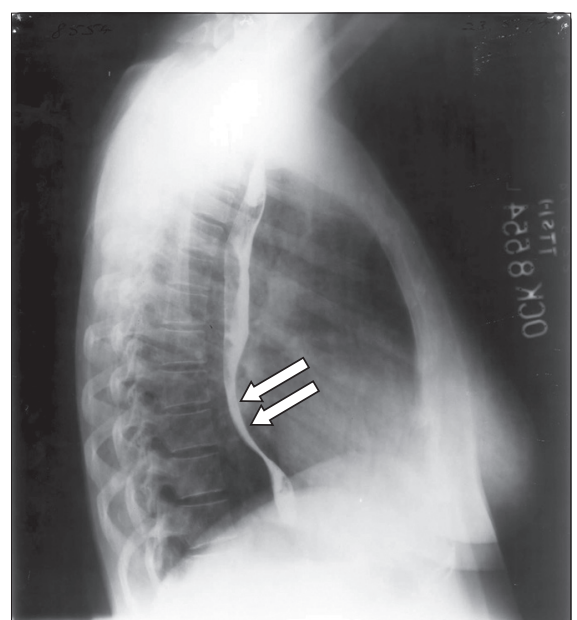

Fig. 4 Lateral projection radiograph shows posterior displacement of the distal oesophagus (arrows) in the setting of left atrial enlargement.

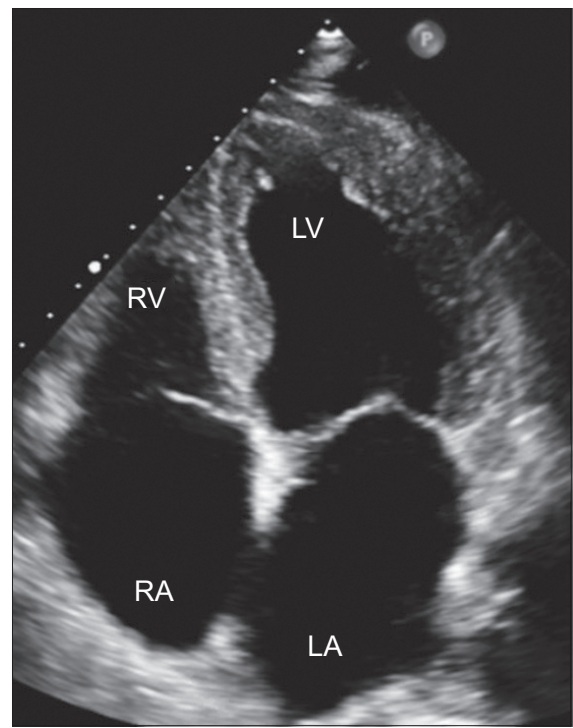

Fig. 5 Transthoracic two-dimensional echocardiography was performed on a middle-aged man (different patient from the earlier patient who underwent a barium swallow procedure) with a history of atrial fibrillation. Echocardiogram in four-chamber view shows both cardiac atria enlarged relative to the ventricles. Note that the appearance of apparent communication between the cardiac atria was due to technical factors during image acquisition, and the patient has no atrial septal defect. LA: left atrium; LV: left ventricle; RA: right atrium; RV: right ventricle

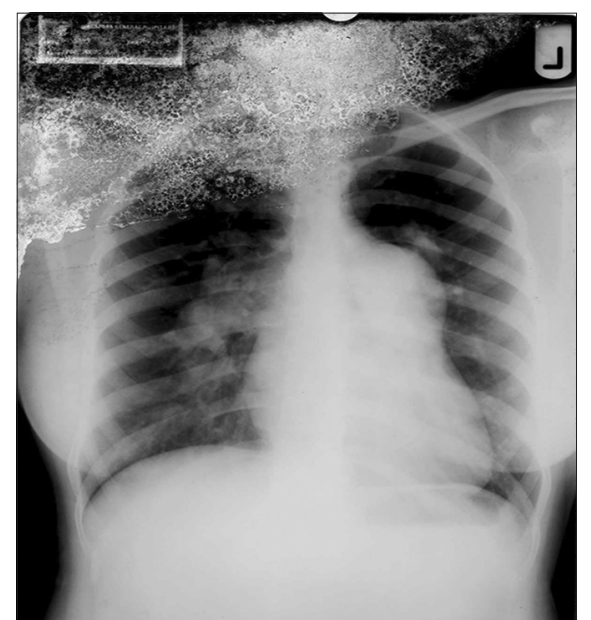

Fig. 6 Chest radiograph of a woman with chronic shortness of breath shows enlargement of the pulmonary trunk and pulmonary arteries with decreased size of the peripheral vessels. Findings are suggestive of the presence of an atrial septal defect with resultant pulmonary arterial hypertension. Film degradation due to ageing is noted.

deoxygenated blood entering the systemic circulation causing cyanosis, termed Eisenmenger syndrome. In Singapore today, this condition can be suspected at the prenatal stage by fetal echocardiography, followed up in the postnatal period, and treated with percutaneous or surgical closure if indicated, decreasing morbidity and mortality.

The upper half of the film (Fig. 6) is degraded due to ageing. X-ray film consists of silver halide emulsion, which undergoes oxidation to produce a silver ion and an electron when exposed to light. Over time, the protective layer of gelatin over the film may be damaged by this oxidative process, as seen in this case. This is no longer seen in the current day due to the advent of digital radiography and the presence of computerised picture archiving and communication systems.

\section{CONJOINED TWINS}

A pair of term dicephalus parapagus conjoined male twins born in the 1980s underwent contrast-enhanced aortography for pre-surgical separation evaluation, which was performed using catheter-guided contrast instillation via the shared umbilical 


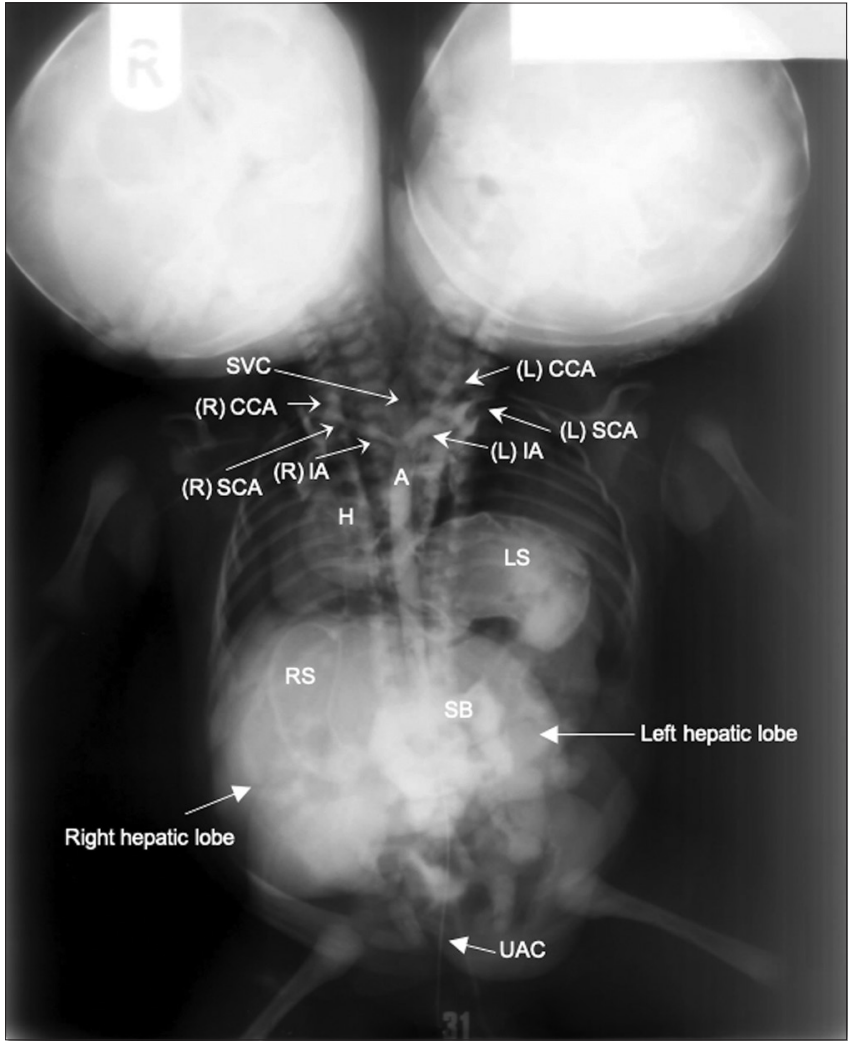

Fig. 7 A pair of term dicephalus parapagus conjoined male underwent catheter-guided contrast instillation via the shared umbilical artery. Contrast-enhanced aortogram shows a single cardiac silhouette in the right hemithorax with a shared common ventricle. A single united thoracoabdominal aorta in the midline provides arterial supply to each twin via the innominate arteries. Cardiovascular anatomy - A: aorta (shared); $\mathrm{H}$ : heart; (R) CCA: right common carotid artery of right twin; (R) IA: right innominate artery (shared); (R) SCA: right subclavian artery in right twin; (L) CCA: left common carotid artery of left twin; (L) IA: left innominate artery (shared); (L) SCA: left subclavian artery in left twin; SVC: superior vena cava, likely shared; UAC: umbilical arterial catheter, tip likely in the left hepatic lobe at the level of L2-3. Other structures - L: liver, shared between both twins, bridging the midline; LS: stomach of the left twin, which shows organoaxial rotation and has herniated through the left hemidiaphragm into the left hemithorax; RS: stomach of right twin; SB: small bowel loops of the left twin, with some loops herniated into the left hemithorax

artery. The study outlines a single cardiac silhouette in the right hemithorax with a shared common ventricle (Fig. 7). A single united thoracoabdominal aorta in the midline provides arterial supply to each twin via the innominate arteries. Conjoined twins present a unique challenge due to complex anatomy involving multiple body systems. Preoperative planning is aided by gleaning pertinent information from a combination of imaging modalities, depending on the site(s) of fusion. ${ }^{(3)}$ In cases of thoracic conjunction, conventional catheter angiography was traditionally performed to define cardiac malformations and aberrant vascular connections. This has now been completely replaced by MR imaging or CT and digital subtraction angiography.

Unfortunately, the depicted case was not amenable to surgical separation given the shared heart. The surgical considerations in the separation of conjoined twins were fraught with ethical considerations and decisions depending on the anatomical configuration encountered, such as considering which twin should receive the pair of lower limbs or the set of sexual organs.

\section{DEEP VEIN THROMBOSIS OF THE LEFT FEMORAL VEIN}

Ascending phlebography was described in the 1970s and was previously the gold standard for the diagnosis of deep venous thrombosis (DVT). It involves cannulation of the dorsal foot veins, and assessment of the profunda vein is done with the Valsalva manoeuvre. Pitfalls of interpretation include false positive examination due to under-filling, dilution or admixture of contrast. ${ }^{(4)}$ In Fig. 8, ascending phlebography shows filling defects and distension of the left femoral vein to the distal femur, in keeping with DVT. The 'tram-track' sign (white arrows) of contrast outlining the incompletely occlusive thrombus is seen. The abrupt transition of the interface between the opacified and non-opacified portion of the femoral vein suggests acute thrombosis (black arrow). The left profunda femoris vein also shows similar evidence of thrombosis (white dashed arrow).

Ultrasonography Doppler assessment has since replaced phlebography for diagnosis of DVT. Nonetheless, this appearance of DVT on venography can still be seen during venous thrombolysis, which is performed to reduce post-thrombotic syndrome.

\section{CHRONIC VENOUS INSUFFICIENCY}

Invasive catheter venography was the gold standard test for venous disease before the introduction of duplex ultrasonography in 1989. ${ }^{(5)}$ Plain radiographs of the calf (Fig. 9) show diffuse periosteal reaction along the tibia and fibula, soft tissue swelling and phleboliths. Ascending catheter venography of the same limb shows prominent superficial calf veins that are consistent with chronic venous insufficiency $(\mathrm{CVI})$. The deep veins are patent.

In the context of suspected $\mathrm{CVI}$, ascending venography evaluates perforator competency and deep venous patency. Contrast is injected into a dorsal foot vein under fluoroscopy with the patient in a $30^{\circ}-45^{\circ}$ foot down position and a tourniquet at the ankle. The tourniquet occludes the superficial veins so that contrast is forced into the deep venous system. Any retrograde flow into perforating veins is diagnostic of perforator incompetence. ${ }^{(5)}$ Descending venography via contrast injection into the common femoral vein grades the anatomic extent of the reflux. ${ }^{(6)}$ Catheter venography remains relevant today if venous reconstruction is contemplated.

\section{SILICOSIS}

Silicosis was the predominant occupational lung disease in Singapore in the 1970s. ${ }^{(7)}$ This was largely attributed to the presence of granite mines such as those in Bukit Timah, Bukit Batok and Pulau Ubin, where workers were exposed to free silica dust from detonating and processing the granite stones. A chest radiograph (Fig. 10) shows the presence of opacities with a hyperdense rim in the bilateral hilar regions (arrows) consistent with calcified lymph nodes. The calcification outlines the peripheries of the hilar nodes and are termed eggshell calcifications. This appearance is suggestive for silicosis given the appropriate setting of occupational exposure, with the differential diagnosis of sarcoidosis (post radiation). 


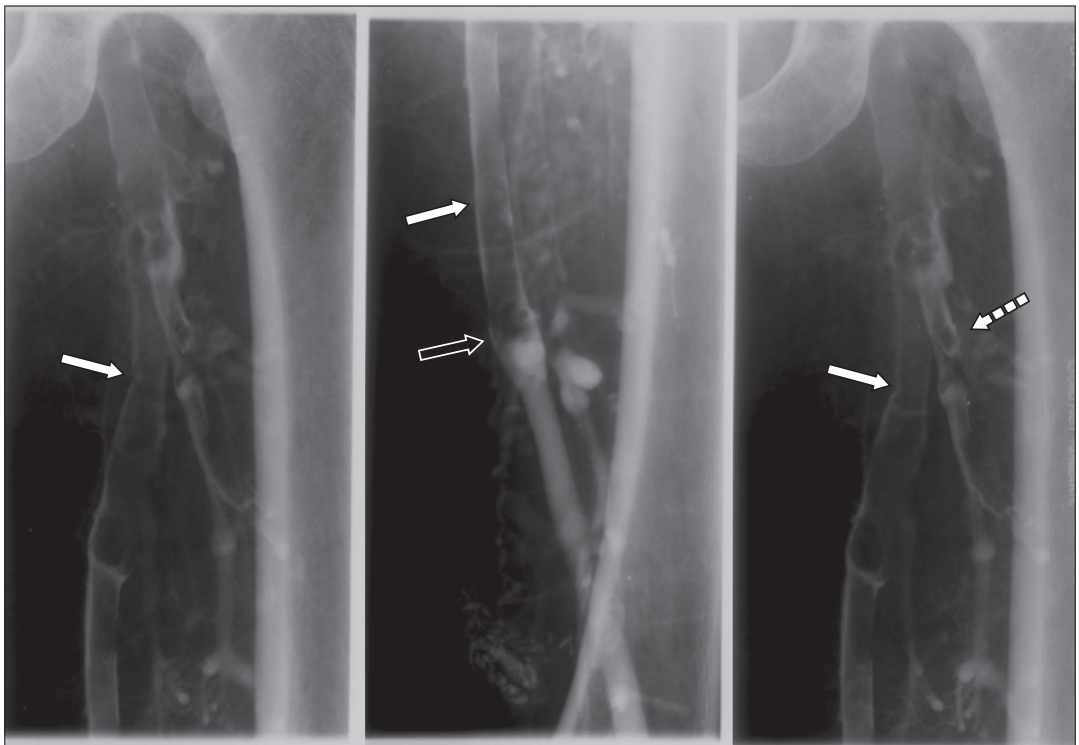

Fig. 8 Ascending phlebogram shows filling defects and distension of the left femoral vein, in keeping with deep venous thrombosis.
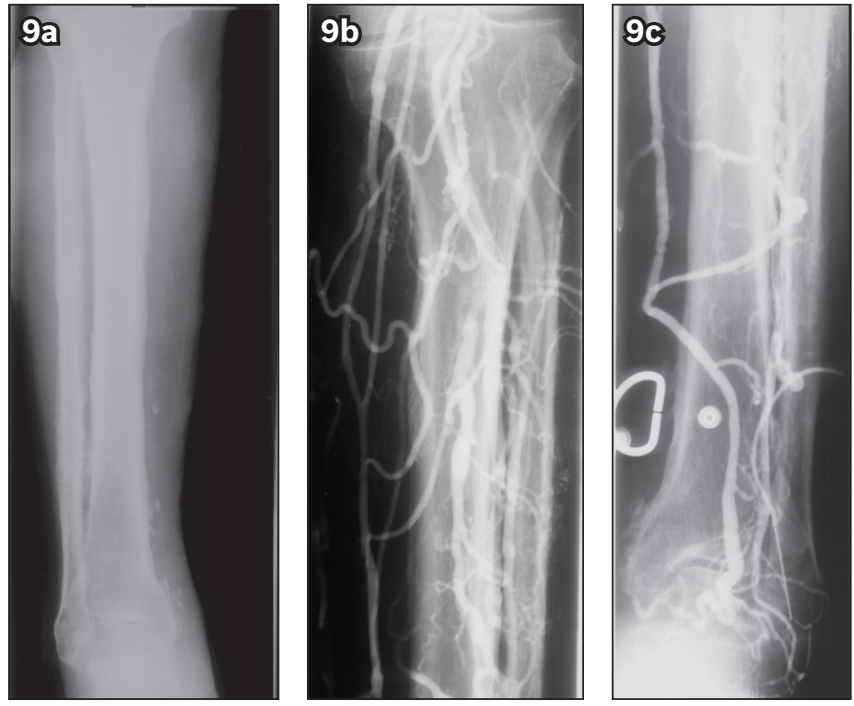

Fig. 9 (a) Plain radiograph of the calf shows diffuse periosteal reaction, soft tissue swelling and phleboliths along the tibia and fibula. (b \& c) Venograms of the same limb show prominent superficial veins. Overall features are consistent with chronic venous insufficiency.

Measures were taken to decrease the prevalence of silicosis. Mines were asked to employ dust control measures. The government necessitated dust exposure monitoring, encouraged use of breathing devices and instituted medical surveillance. In the late 1990s, granite mining licensing was reduced and eventually stopped. Other industries associated with development of silicosis in its workers included the rubber industry, kaolin mines, foundries and brickworks. In the current age, as seen in Fig. 11, CT shows superior characterisation of mediastinal lymph node calcifications compared to radiography (asterisk). CT also better demonstrates the presence of pulmonary nodules in the upper lobes forming conglomerate focal masses, some sausage-shaped, with irregular margins and adjacent emphysematous changes (arrows), compatible with a diagnosis of complicated pneumoconiosis secondary to silicosis.

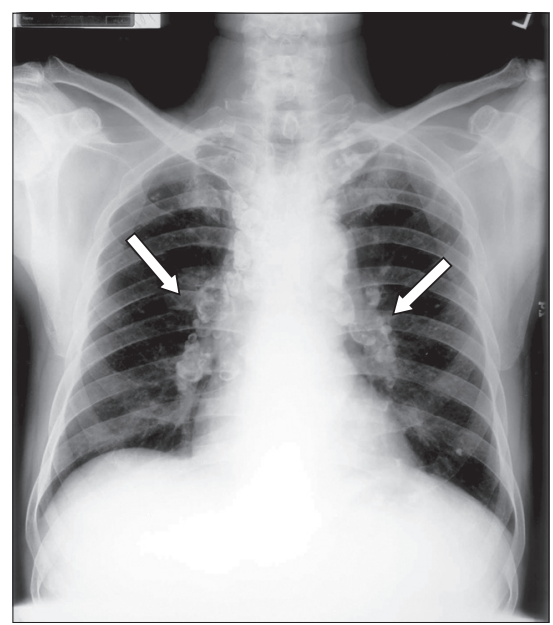

Fig. 10 Chest radiograph shows imaging features of bilateral hilar adenopathy demonstrating an eggshell calcification pattern (arrows), seen in the setting of silicosis.

\section{ASBESTOS LUNG DISEASE}

From the 1950s to 1980s, raw asbestos was imported and mixed with cement in a factory to produce roof tiles, ceiling boards and pipes. It was also used as insulating material in shipyard industries and buildings as well as friction material for heavy vehicle components. The chest radiograph of an asymptomatic factory worker (Fig. 12) showed curvilinear calcifications along both hemidiaphragms and irregular calcifications in the bilateral lower zones (arrows), representing pleural calcifications. The multiplicity, bilaterality and diaphragmatic involvement are pathognomonic for asbestos plaques rather than other differentials such as pleural masses, rib fractures, prior empyema, tuberculosis and haemothorax.

During the 1980s, as the association between asbestos and lung disease became apparent, its use was curtailed. Asbestos was completely banned for use in 2001. Occupational asbestos exposure is, however, still seen today, mainly during demolition or renovation of buildings constructed before 1989.(8) Under the Workplace Safety and Health Regulations 2011, chest 


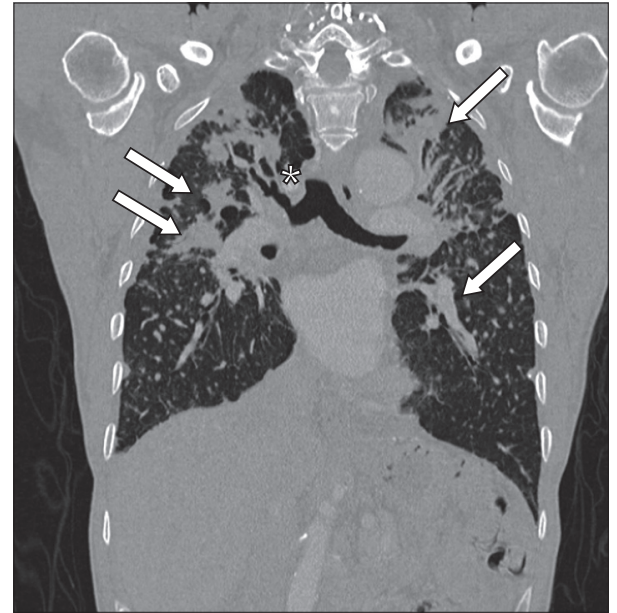

Fig. 11 CT image of a patient with complicated pneumoconiosis secondary to silicosis. Note the superior characterisation of mediastinal lymph node calcifications on CT compared to radiography (asterisk).

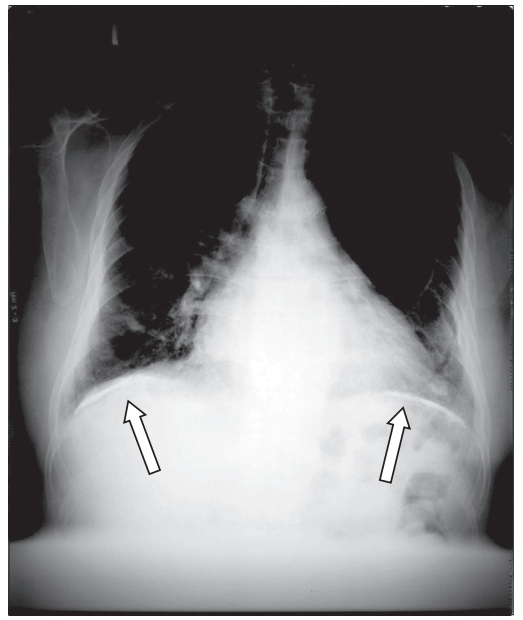

Fig. 12 Chest radiograph of an asymptomatic factory worker shows curvilinear calcifications along both hemidiaphragms (arrows) representing pleural calcifications. Asbestos lung disease must be considered in the imaging differential.

radiography is to be performed every 36 months for workers with asbestos exposure.

\section{EMERGENCY MYELOGRAM IN ACUTE PARAPLEGIA}

A patient with a history of oesophageal carcinoma presented with acute paraplegia, and emergent myelography was performed. The fluoroscopy-guided myelogram (Fig. 13) demonstrates a filling defect within the anterior aspect of the column of contrast within the spinal canal (arrow) secondary to local invasion by the tumour.

Myelography was first described in 1921. This procedure involved intrathecal instillation of contrast under fluoroscopic guidance by a radiologist, followed by the acquisition of fluoroscopic and, in later years, CT images of the spinal canal. The first contrast medium used was oil-based Myodil, which was used for years and later found to cause arachnoiditis. It was replaced when non-ionic water soluble contrast media became available in the 1980s.

For decades, myelography was the only diagnostic method available to investigate pathology within the spinal canal until the

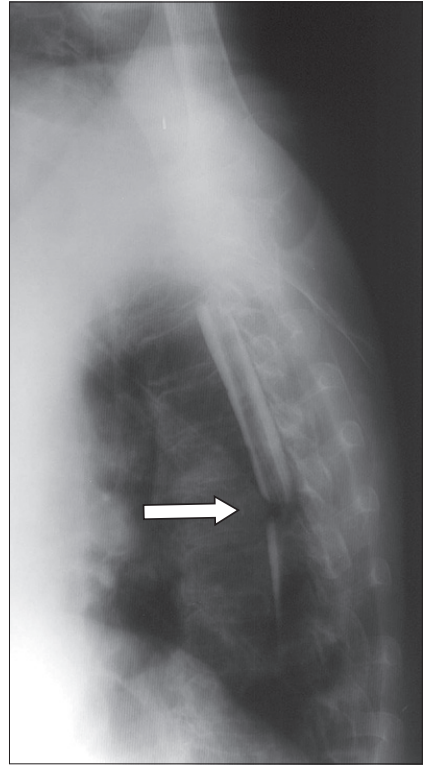

Fig. $13 \mathrm{~A}$ patient with a history of oesophageal carcinoma presented with acute paraplegia. Emergent fluoroscopy-guided myelogram shows a filling defect within the anterior aspect of the column of contrast within the spinal canal (arrow) secondary to local invasion by the tumour.

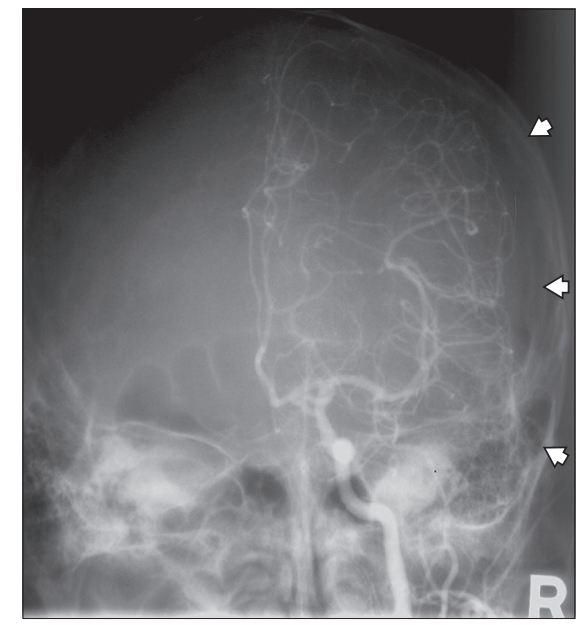

Fig. 14 Catheter cerebral angiogram of the right internal carotid artery shows leftward deviation of the branches of the anterior and middle cerebral arteries across the midline of the skull with a clear crescentic avascular space under the skull (arrowheads). These findings are worrisome for the presence of a subdural haematoma; this diagnosis was subsequently confirmed on right craniectomy.

advent of MR imaging in the late 1980s. At present, myelography is still performed in patients for whom MR imaging is not possible for safety reasons ${ }^{(9)}$ (e.g. non-MR-compatible pacemakers), and in patients with metal spinal implants that would result in severe degradation of MR image quality due to artefacts.

\section{SUBDURAL HAEMATOMA}

Catheter cerebral angiography of the right internal carotid artery was performed in a patient with left hemiplegia (Fig. 14). The angiogram demonstrates leftward deviation of the branches of the anterior and middle cerebral arteries across the midline of the skull with a clear crescentic avascular space under the skull. These findings are consistent with an extra-axial mass lesion adjacent to the right cerebral hemisphere, likely representing 


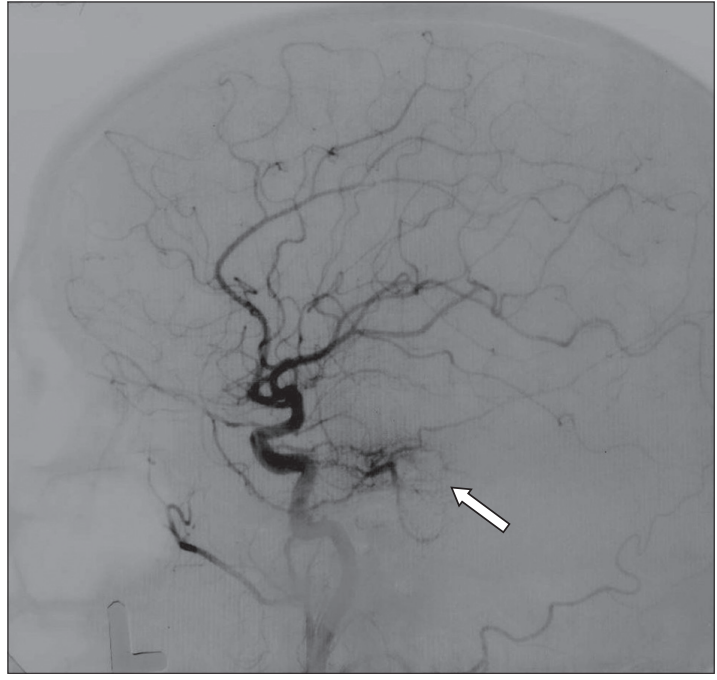

Fig. 15 Angiogram of the left internal carotid artery shows an abnormally prominent meningohypophyseal artery arising from the cavernous segment of the left internal carotid artery and leading to a region of abnormal seeming blood vessels, which show a characteristic 'tumour blush' (arrow) angiographic pattern. Surgery was performed, which demonstrated tentorium cerebelli meningioma.

a subdural haematoma (arrowheads). The diagnosis was subsequently confirmed on right craniectomy in which a blood clot was evacuated.

Prior to the advent of routine head CT imaging, emergent catheter cerebral angiography was the default radiological investigation for patients with neurological symptoms. ${ }^{(10)}$ This study was performed at TTSH, which was the first hospital designated in Singapore for specialised neurosurgical procedures. The investigation, performed via femoral catheter or direct arterial puncture, was used to differentiate the pathological processes of intracranial mass (tumour blush pattern or deviation of vascular structures), infarct (absence of blood flow) and intracranial haemorrhage (vascular blush pattern in intraparenchymal haemorrhage, or the presence of an irregularly contoured intracranial aneurysm with or without mass effect in ruptured aneurysmal subarachnoid haemorrhage). Hydrocephalus may also be suspected and confirmed by air encephalography or ventriculography.

\section{MENINGIOMA WITH PROMINENT MENINGOHYPOPHYSEAL ARTERY}

Urgent catheter cerebral angiography of the left internal carotid artery was performed for a patient who presented with right hemiparesis (Fig. 15). No vascular occlusion was detected. However, there was a prominent artery arising from the cavernous segment of the left internal carotid artery, running posteriorly and terminating at a localised collection of capillaries with adjacent contrast staining (arrow). The location, course and size of this vessel is in keeping with a prominent meningohypophyseal artery $^{(11)}$ leading to a region of abnormal appearing blood vessels which show a characteristic 'tumour blush' angiographic pattern. The normal meningohypophyseal artery is often too small to be visualised on angiography. When it is seen to be prominent, a pathological process should be considered, for example, the presence of a tumour at the tentorium cerebelli, which is the region this vessel supplies. The patient underwent surgery and a tentorium cerebelli meningioma was found intraoperatively and excised. CT and MR imaging brain studies have since superseded catheter angiography for the diagnosis and localisation of intracranial lesions.

\section{ACKNOWLEDGEMENTS}

The authors would like to acknowledge the staff of the Department of Diagnostic and Interventional Imaging, KK Women's and Children's Hospital, Singapore, for their assistance in digitising the hard copy films from the SingHealth Radiology Archives; Dr Kamalesh Anbalakan (Cardiology) for the two-dimensional echocardiography image on the case 'left atrial enlargement'; and Dr David Alexander Stringer (Paediatric Radiology) for his input on the case 'conjoined twins'.

\section{REFERENCES}

1. Syme J. Transfemoral catheter aortography in dissecting aneurysm. Australas Radiol 1971; 15:133-9.

2. Gotsman I, Mogle P, Shapira MY. An unusual cause of dysphagia. Postgrad Med J 1999; 75:629-31.

3. Kingston CA, McHugh K, Kumaradevan J, Kiely EM, Spitz L. Imaging in the preoperative assessment of conjoined twins. Radiographics 2001; 21:1187-208.

4. Thomas ML. Phlebography. Arch Surg 1972; 104:145-51.

5. van Bemmelen PS, Bedford G, Beach K, Strandness DE. Quantitative segmental evaluation of venous valvular reflux with duplex ultrasound scanning. J Vasc Surg 1989; 10:425-31.

6. Kistner RL. Diagnosis of chronic venous insufficiency. J Vasc Surg 1986; 3:185-8.

7. Lee HS, Phoon WH, Wang SY, Tan KP. Occupational respiratory diseases in Singapore. Singapore Med J 1996; 37:160-4.

8. Lim JW, Koh D, Khim JSG, Le GV, Takahashi K. Preventive measures to eliminate asbestos-related diseases in Singapore. Saf Health Work 2011; 2:201-9.

9. Ozdoba C, Gralla J, Rieke A, Binggeli R, Schroth G. Myelography in the age of MRI: why we do it, and how we do it. Radiol Res Pract 2011; 2011:329017.

10. Lee Ligon B. Biography: history of developments in imaging techniques: Egas Moniz and angiography. Semin Pediatr Infect Dis 2003; 14:173-81.

11. Pribram HF, Boulter TR, McCormick WF. The roentgenology of the meningo-hypophyseal trunk. Am J Roentgenol Radium Ther Nucl Med 1966; 98:583-94 


\section{SINGAPORE MEDICAL COUNCIL CATEGORY 3B CME PROGRAMME} (Code SMJ 202012B)

Question 1. Regarding aortic dissection:

(a) A Stanford Type A dissection arises proximal to the left subclavian artery.

(b) The true lumen is of lower radiographic density than the false lumen.

(c) The presence of a dissection flap is a sign of aortic dissection on computed tomography (CT) angiography.

(d) CT angiography has superseded catheter aortography in the diagnosis of aortic dissection.

Question 2. Regarding left atrial enlargement:

(a) A barium swallow performed in the lateral position in the setting of left atrial enlargement shows posterior displacement of the distal oesophagus.

(b) The left atrium is the most anteriorly located cardiac chamber.

(c) Mitral valve disease can cause left atrial enlargement.

(d) Patients with this condition can present with hoarseness of voice due to Ortner syndrome.

Question 3. Classic radiological features of silicosis include:

(a) Calcified lymph nodes.

(b) Pulmonary nodules.

(c) Ground-glass changes.

(d) Cystic lymph nodes.

Question 4. Regarding Eisenmenger syndrome:

(a) It is a sequela of chronic pulmonary arterial hypertension.

(b) It may be associated with an atrial septal defect.

(c) It is associated with cyanosis due to a right-to-left shunt.

(d) It is associated with right ventricular hypertrophy.

Question 5. Regarding catheter cerebral angiography:

(a) In the presence of an intracranial mass lesion, the vascular structures will deviate towards the side of the lesion.

(b) A focal collection of capillaries with adjacent contrast staining is present in an acute infarct.

(c) It remains the gold standard method for the characterisation of intracranial aneurysms.

(d) Its role as the default radiological investigation for patients with neurological symptoms has since been superseded by CT and MR imaging.

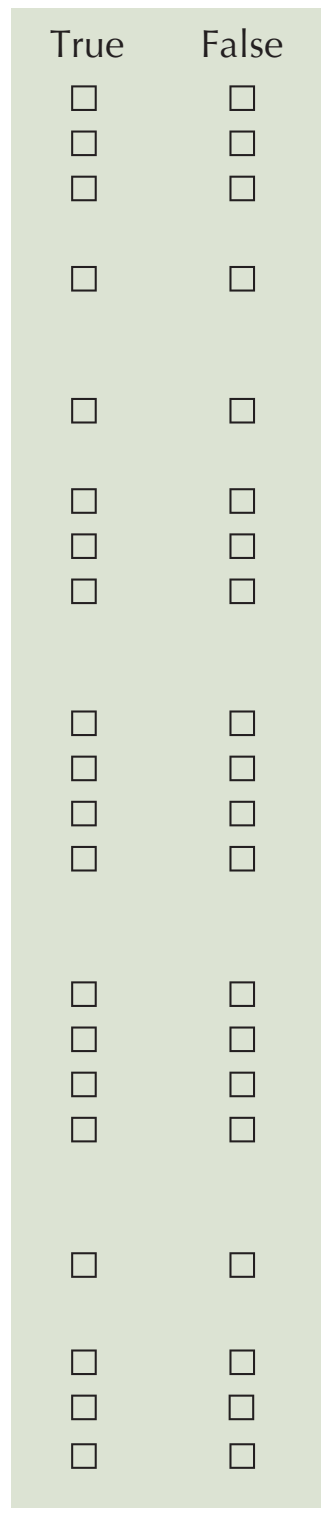

\section{Doctor's particulars:}

Name in full:

Specialty:
MCR no.

Email:

\section{SUBMISSION INSTRUCTIONS}

Visit the SMJ website: http://www.smj.org.sg/current-issue and select the appropriate quiz. You will be redirected to the SMA login page.

For SMA member: (1) Log in with your username and password (if you do not know your password, please click on 'Forgot your password?'). (2) Select your answers for each quiz and click 'Submit'.

For non-SMA member: (1) Create an SMJ CME account, or log in with your SMJ CME username and password (for returning users). (2) Make payment of SGD 21.40 (inclusive of $7 \%$ GST) via PayPal to access this month's quizzes. (3) Select your answers for each quiz and click 'Submit'.

RESULTS:

(1) Answers will be published online in the SMJ February 2021 issue. (2) The MCR numbers of successful candidates will be posted online at the SMJ website by 8 February 2021. (3) Passing mark is $60 \%$. No mark will be deducted for incorrect answers. (4) The SMJ editorial office will submit the list of successful candidates to the Singapore Medical Council. (5) One CME point is awarded for successful candidates. (6) SMC credits CME points according to the month of publication of the CME article (i.e. points awarded for a quiz published in the December 2020 issue will be credited for the month of December 2020, even if the deadline is in February 2021).

Deadline for submission (December 2020 SMJ 3B CME programme): 12 noon, 1 February 2021. 